\title{
Spatial judgments of exocentric extents in an open-field situation: Familiar versus unfamiliar size
}

\author{
JOHN PREDEBON \\ University of Sydney, Sydney, New South Wales, Australia
}

\begin{abstract}
Carlson and Tassone (1971) found that an object of familiar size presented in an outdoor setting and viewed at an appreciable distance is judged to be more distant than an unfamiliar object. Three experiments examined whether object familiarity also affects spatial judgments of exocentric extents presented under conditions comparable to those of Carlson and Tassone's experiments. The markers of the extents were either familiar or unfamiliar objects. In Experiment 1, subjects reproduced the perceived extents of depth intervals by adjusting a comparison egocentric extent, and in Experiment 2, subjects also compared the relative depths of two equally long extents. In Experiment 3, the two equally long extents were presented in the subjects' frontoparallel plane, and the subjects reported which of these two extents appeared longer and farther away. The results of these experiments indicate that familiar size does not affect the perceived depths or lengths of exocentric extents viewed under naturalistic conditions.
\end{abstract}

The issue of whether or not familiar size influences perceived distance has been investigated mainly under conditions of cue reduction-that is, under conditions in which other cues to distance are eliminated or their effectiveness is attenuated (see Sedgwick, 1986). In contrast, only a few studies (Carlson \& Tassone, 1971; Fillenbaum, Schiffman, \& Butcher, 1965; Predebon, 1979a, 1979b, 1990) have evaluated the effect of familiar size on perceived distance under naturalistic, open-field viewing conditions.

Carlson and Tassone (1971) found that at an appreciable distance a normal-sized familiar object (a person) is judged to be farther away than an unfamiliar object (a board), and similar results have been obtained by Predebon (1990), who used two different indicators of perceived distance. Carlson and Tassone (1971) proposed that this effect could be explained in terms of a change in the subjective distance scale caused by the presence of a familiar object in the visual field. Specifically, the subjective magnitude of a given unit of physical distance becomes increasingly smaller at progressively greater distances from the observer. While the introduction of an unfamiliar object in the field does not affect the distance scale established by the distance information (e.g., texture, perspec-

This research was funded by Australian Research Council Grant A78930099. The data of Experiment 1 were collected by M. Fox, S. Gallart, L. Luck, G. Miller, T. Poore, C. Saunders, J. Sinclair, S. Sturevska, and J. Tombelson, in partial fulfillment of the requirements of the M.A. (Pass) degree. I am grateful to Very Rev. L. G. Cashen, Rector, St. John's College, for permitting Experiment 1 to be conducted on the college grounds. Correspondence should be addressed to John Predebon, Department of Psychology, University of Sydney, Sydney, New South Wales 2006, Australia. tive) already present, a familiar object will result in a rescaling of perceived distance as a consequence of the visual metric of the familiar object. The more distant physical units near the familiar object will be rendered more equal in subjective magnitude to nearby units, and as a result, the familiar object will be perceived to be farther away than an unfamiliar object.

The purpose of the present investigation was to determine the generality of Carlson and Tassone's (1971) effect, because of the effect's obvious significance for certain theoretical treatments of space perception. Specifically, it challenges theories (e.g., Gibson, 1950) that reject any significant contribution of past experience in determining perceptions of spatial extents. If, under naturalistic viewing conditions, familiar size is demonstrated to reliably and systematically influence a variety of spatial tasks, credence is given to the claim that familiar size is a significant perceptual datum of experience that needs to be taken into account by any comprehensive theory of space perception.

Carlson and Tassone (1971) and Predebon (1990) examined the influence of familiar size on judgments of the relative perceived egocentric distances of familiar and unfamiliar objects: Subjects either reported which of the two objects appeared farther away (Carlson \& Tassone, 1971; Predebon, 1990) or adjusted the egocentric distance of the familiar object to match the egocentric distance of the unfamiliar object (Predebon, 1990). The purpose of the three experiments reported here was to determine whether Carlson and Tassone's (1971) effect would also occur for exocentric extents-that is, to determine whether or not judgments of the depth or length of an exocentric extent viewed under naturalistic, open-field conditions are affected by the familiarity or otherwise of the objects that 
demarcate the exocentric extent. Assuming the sizedistance invariance hypothesis (Ittelson, 1960), the rescaling effect of familiar size on the subjective distance scale should increase not only the perceived distance of a familiar object but also the perceived extent of intervals demarcated by familiar objects. For example, if familiar size increases the perceived distance of an object by a factor of two, the depth between two identical familiar objects will also increase by the same factor. Experiment 1 required subjects to match a comparison egocentric distance to the perceived depth of extents that were demarcated either by familiar (person) or unfamiliar (board) markers. Experiment 2 was similar to Experiment 1, except that subjects also compared the relative depths of two intervals, one demarcated by persons and the other by boards, presented at the same egocentric distance but at different radial directions from the subject. In Experiment 3, the exocentric extents were orthogonal to the subject's line of sight, and the task was to report the perceived relative lengths and distances of the extents.

\section{EXPERIMENT 1}

Experiment 1 was designed not only to determine the effect of familiar size on judgments of depth, but also to identify the minimum egocentric distance of the depth interval at which the effect occurs. According to Carlson and Tassone's (1971) hypothesis, the rescaling effect of familiar size on perceived distance and, hence, on perceived depth is more likely to occur at far viewing distances.

\section{Method}

Subjects. The subjects were 40 volunteer students from the University of Sydney. All subjects had normal or corrected vision.

Test site and stimuli. The experiment was conducted on the grounds of St. John's College, University of Sydney. The test field was a level grassy area approximately $40 \mathrm{~m}$ wide and $280 \mathrm{~m}$ long. Relative to the subject's location, the right-hand side of the test area was flanked by two adjoining football fields each containing pairs of goal posts, and on the left-hand side the ground sloped gently upward toward the grounds and buildings of a university residential college. The far end of the test area was flanked by shrubs and a boundary hedge beyond which could be seen commercial buildings and the passing traffic of a busy arterial road. Beyond the football fields and behind and to the right of the subject's location could be seen shrubs and trees and a boundary fence. Occasionally, people could be seen on the grounds of the college, and traversing the football fields.

The depth intervals were demarcated by either two men or two boards. The men, each approximately $180 \mathrm{~cm}$ tall and of similar weight, wore white laboratory coats and a white head scarf. They stood facing the subject with their hands behind their backs. The boards, painted flat white, were $185 \mathrm{~cm}$ tall and $44 \mathrm{~cm}$ wide. Each board, with its long axis positioned vertically with respect to gravity, was held in place by a person who hid behind the board.

There were three depth intervals $(15,30$, and $45 \mathrm{~m})$, each of which was presented at each of three distances, with the near marker of the interval $10,87.5$, or $165 \mathrm{~m}$ from the subject's location. To avoid overlap of the far marker by the near marker, relative to the line of sight from the subject's location point and the near marker, the far marker was displaced slightly to the right of the near marker.
The comparison field was located approximately $90^{\circ}$ to the right of the subject's line of sight of the depth intervals in the test field. The comparison target was either a person dressed in a white laboratory coat or a white painted board, $185 \mathrm{~cm}$ tall and $44 \mathrm{~cm}$ wide, held vertically by a person behind the board. The maximum extent of the comparison field from the subject's location was approximately $80 \mathrm{~m}$. Reference points were placed at $5-\mathrm{m}$ intervals along the comparison field. These were not visible to the subject and were used to measure the subject's reproductions of the depth of the intervals in the test field.

Design and Procedure. There were two conditions, with 20 subjects in each. In one condition, the comparison target and the depth interval markers were persons, and in the other condition they were boards.

The subjects were tested individually. They were met at a specified location and escorted to the test site. The task was to reproduce the actual distance between the two markers in the test field by "adjusting" the distance of the comparison target from the subject's location. The instructions specified judgments of the actual or objective depth of the interval-that is, "the distance you would obtain if you were to measure the distance between the two boards with a tape measure." On all trials, the initial position of the comparison target was set at $40 \mathrm{~m}$ from the subject's location. ${ }^{1}$ The subjects were required to indicate the direction in which the comparison target was to move and to signal "stop" when the target's distance matched the actual depth of the test extents. The person comparison target and the person behind the board moved at a steady pace along a straight line, either backward from or forward to the subject. The experimenter vocally communicated the subject's directions to the comparison target. Bracketing was permitted, and the subjects were allowed to look back and forth between the test and comparison fields until they were satisfied with their judgments. After completing a judgment, the subject turned away from the test and comparison fields. The experimenter then communicated to the person markers in the test field, via walkie-talkie transmission if necessary, to move to their predetermined positions for the next trial. During this time the comparison-target person measured the distance from the tip of his shoes to the nearest reference point in the comparison field. After the comparison target moved to the $40-\mathrm{m}$ starting position and the markers positioned themselves at the appropriate spots in the test field, the subject turned around and the procedure was repeated.

The subjects made a total of nine judgments of depth, one judgment of each of the three depth intervals at each of the three egocentric distances. All subjects completed the three judgments at one egocentric distance before proceeding to the three depth intervals at the next egocentric distance. However, the order of presentation of the three depth intervals was randomized, as was the order of presentation of the three sets of egocentric distance depth intervals.

\section{Results}

Each subject's depth match was converted to a signed error score, in meters, by subtracting the actual length of the depth interval from the depth-matching judgment, with positive and negative errors indicating over- or underestimation, respectively, of the actual length of the interval. A three-way analysis of variance (ANOVA), with repeated measures on the depth interval and egocentric distance factors and with marker type as the betweensubjects factor, was performed on these data. The marker type factor was not significant either as a main effect or in interaction with the other two factors. Therefore, the results of the two marker-type conditions were averaged, and the mean errors of the resulting nine combined conditions are shown in Figure 1. 


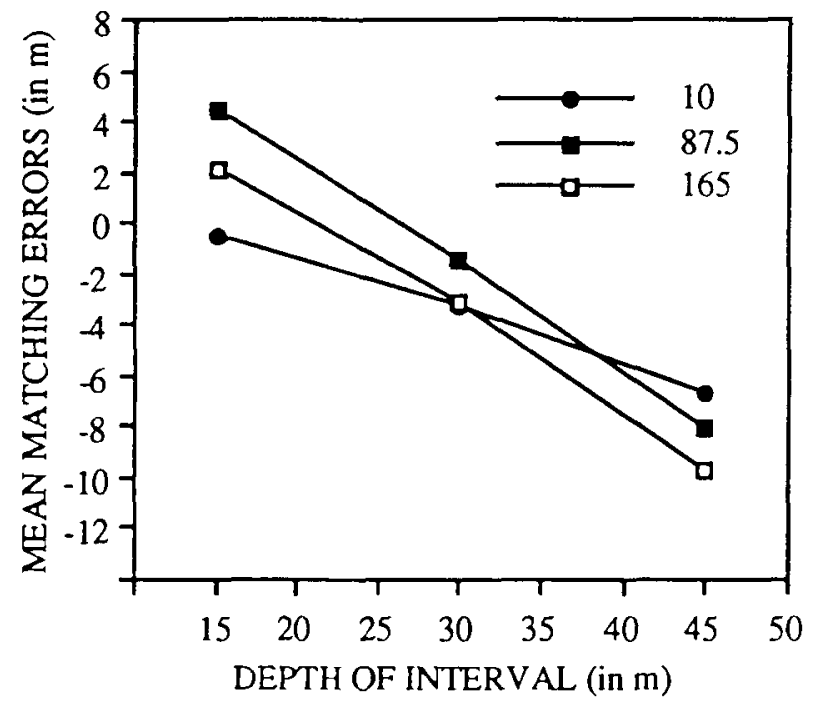

Figure 1. Mean matching errors, in meters, averaged over depth marker type, for the three depth intervals $(15,30$, and $45 \mathrm{~m})$ at each of the three egocentric distances $(10,87.5$, and $165 \mathrm{~m})$ in Experiment 1 .

The main effect of depth interval was significant $[F(2,76)=107.4, p<.05]$, indicating, as is shown in Figure 1, that the magnitude of the errors differed across the three depth intervals. Whereas the $15-\mathrm{m}$ depth interval tended to be overestimated, the 30-m interval tended to be underestimated and the 45-m interval even more so. The only other significant effect was the interaction between the egocentric distance factor and the depth-interval factor $[F(4,152)=10.1, p<.05]$, and the nature of this interaction is evident in Figure 1.

\section{Discussion}

The major result of significance is the failure of marker type to influence judgments of depth. Although this suggests that familiar size does not influence the perceived depth of extents, other explanations need to be considered. First, the repeated measures design may have masked the expected interaction between the effectiveness of familiar size and the egocentric distance of the depth intervals. Specifically, since it is unlikely that familiar size will alter the subjective distance scale at the near egocentric distances, exposure to the depth intervals at the 10- and 87.5-m distances may have given subjects an indication of the actual depths of the intervals at the 165 -m distance. This possibility can be excluded, however, by considering the depth judgments of the 7 subjects in each condition who were presented with the 165 -m depth intervals first. The results of the two-way ANOVA with depth interval as the repeated measures factor and marker type as the between-subjects factor yielded a significant effect of depth interval $[F(2,24)=18.02, p<.05]$, indicating that the magnitude of the errors differed across the three depth intervals. Importantly, however, neither the main effect of marker type nor the interaction between the marker-type and the depth-interval factors was significant, indicating that familiar size did not influence the depth matches of the $165-\mathrm{m}$ intervals.

Second, the null effect of marker type might be attributed to the subjects' having acquired an assumed or familiar size of the board markers. The physical-size identity of the comparison and marker boards, together with the relative proximity of the comparison board to the subject's location, led subjects to correctly infer or assume the metric size of the marker boards, in which case the present findings can be explained in terms of the equivalent effects of the person and board markers on the subjective distance scale.

Third, the objective-distance instructions used here raise a potential complication. Gogel (1973) has argued that since observers frequently perceive a depth interval at a far distance as shorter than a same interval at a near distance, then, as a consequence of this experience, they are likely to apply a correction to their reports of distance in an attempt to give a correct judgment. Furthermore, it seems reasonable to suppose that such a cognitive correction to reports of distance is more likely to occur with objective- than with apparent-distance instructions (Gogel, 1973). In agreement with this possibility, Rogers and Gogel (1975) found that with a bisection task, apparentdistance instructions tended to produce lower values of the exponent of the power function relating judgments of distance to physical distance than did objective-distance instructions. Conceivably, in the present experiment the tendency to cognitively correct reports of distance may have masked any effect of familiar size on perceived depth.

Finally, it is possible that the person markers induced a nondetectable change in the perceived distance scale. Given the subjects' possible exposure to, and hence familiarity with, the football fields and university buildings, the person interval markers are unlikely to have caused a significant change in the perceived distance scale to that already effected by the adjacent football fields and college buildings. This possibility implies that Carlson and Tassone's (1971) original finding might not occur under the conditions prevailing in Experiment 1 . In their experiments, the familiar (person) and unfamiliar (board) objects were located $77^{\circ}$ apart, and both were $183 \mathrm{~m}$ from the subject's location. Since the physical constraints of the test site made it impossible to duplicate Carlson and Tassone's stimulus conditions, the aim of Experiment 2 was to determine the effect of familiar size on judgments of depth under conditions in which a prior study (Predebon, 1990) had replicated their finding.

\section{EXPERIMENT 2}

This experiment assessed the effects of familiar size on two different judgments of depth-namely, the depthmatching task of Experiment 1 and a relative depth task in which subjects compared the depths of two equally long intervals, both of which were presented at the same 
egocentric distance but in different radial directions from the subject's location. The relative depth task was similar to Carlson and Tassone's (1971) task, except that single familiar and single unfamiliar objects were replaced by exocentric depth extents.

\section{Method}

Subjects. The subjects were 26 volunteer undergraduate students, all of whom had corrected or normal vision.

Test site, Stimuli, and Design. The test site, a public park adjacent to the main university campus, has been described in detail elsewhere (Predebon, 1990). The two depth intervals were located approximately $44^{\circ}$ apart, with the near marker $160 \mathrm{~m}$ from the subject's location. The far marker was positioned $15 \mathrm{~m}$ behind the near marker. To avoid overlap of the far and near markers, the far marker was displaced slightly to the right of the near marker. The markers for one of the intervals were two women, approximately $170 \mathrm{~cm}$ tall and of similar weight. The women wore white laboratory coats and white head scarves, and they stood erect facing the subject's location with hands behind their backs. For the other interval, the markers were two white boards, $170 \mathrm{~cm}$ high and $30 \mathrm{~cm}$ wide, held vertically by supports invisible to the subjects. The test area was flanked on the left and on the upper right by open space and a few large trees and shrubs, and on the lower right by a brick building. Beyond the test area could be seen the boundary fence against a backdrop of university buildings. In the test area there were several trees, lampposts, and park benches, and a small paved access road, $3.5 \mathrm{~m}$ wide, crossed the test area approximately $15 \mathrm{~m}$ in front of the subject's location. The ground sloped gently upward away from the subject's location, and the subject had unimpeded views of the depth intervals.

A comparison field was used in the first phase of the experiment. This field was a level area located approximately $67^{\circ}$ to the left of the left-hand depth interval in the test area. A woman wearing a white laboratory coat served as the comparison target.

Procedure. Each subject was escorted to the test location and tested individually. In the first phase of the experiment, the subjects were positioned at the observation point facing the comparison field. The apparent instructions specified judgments based on visual impressions: the subjects were told that their task was to judge the apparent depth of a series of depth intervals (in fact, only one was presented) by matching the distance of the comparison person to the perceived or apparent distance between the markers of the depth interval in the test field. The subjects then turned to view the depth interval and made their judgments. In response to the subject's commands, the comparison target moved at a steady pace either toward or away from the subject's location until the subject signaled "stop." There were two trials with two different initial starting positions of the comparison target, 5 and $25 \mathrm{~m}$ from the subject's location. After completing each judgment, the subject turned around while the comparison person measured and recorded the distance between the tip of her shoes and reference markers in the comparison field. For half the subjects, the depth interval was demarcated by persons; for the other half, by the boards.

In the second phase of the experiment, the stimuli were the two depth intervals presented $44^{\circ}$ apart. The subjects turned to face the test area and were asked to nominate which of the two depth intervals "gives the visual impression of looking longer." The left/right locations of the depth intervals were controlled across subjects. The subjects then turned away from the test site and waited for the third phase of the experiment.

In the third phase, the test objects were a single person and a single board. These were located at the positions of the near markers of the stimuli used in the second phase of the experiment; that is, they were $44^{\circ}$ apart and $160 \mathrm{~m}$ from the subject's location. First the subjects were asked to judge which of the two objects "gives the visual impression of looking taller/smaller"; this was followed by a judgment of which of the two objects appeared farther/closer. For the last two phases of the experiment, equality judgments were not permitted.

\section{Results}

Phase 1. Each subject's two reproductions of the apparent depth of the test interval were averaged and subtracted from the interval's actual distance $(15 \mathrm{~m})$ to yield a single score for that subject. The means, with standard errors in parentheses, of these scores were $10.5 \mathrm{~m}$ (1.68) and $12.4 \mathrm{~m}(1.48)$ for the two groups of subjects judging the depth of the person and board intervals, respectively. The difference between these means is not significant $[t(24)=.87, p>.05]$, indicating that familiar size does not influence judgments of depth.

Phase 2. When asked to compare the relative extents of two depth intervals, 11 of the 26 subjects nominated the interval demarcated by the persons as longer than the interval demarcated by the boards. This proportion (.42) is not significantly different from .5 .

Phase 3. In comparing the apparent size and distance of a single person relative to a single board, 19 and 26 of the 26 subjects reported the person as smaller in apparent size and more distant than the board, respectively. These proportions $(.73,1.0)$ are significantly different from .5 .

The results of Phase 3 of the experiment confirm Carlson and Tassone's (1971) finding that a familiar object is judged to be more distant and smaller in apparent size than an unfamiliar object presented at the same egocentric distance as the person. In contrast, both the depthmatching task and the relative depth task indicate that familiar size does not influence judgments of the perceived extent of a depth interval.

The present findings can be interpreted as indicating that familiar size, although influencing judgments of the apparent relative size and distance of objects, either is not involved or plays only a minor role in discriminations of depth. If depth discriminations are determined mainly by, say, the relative heights of the interval markers in the visual field or on the relative proportions of the background texture occluded by the interval markers, the failure of familiar size to affect judgments of relative depth is unsurprising. Arguably, since Carlson and Tassone's (1971) hypothesis was formulated to account for the influence of familiar size on perceptions of relative egocentric distance, a more appropriate test of the effect of familiar size on judgments of the apparent extent of exocentric intervals is to assess the influence of familiar size on perceptions of the relative lengths and relative egocentric distances of exocentric extents presented in the subject's frontoparallel plane. In this case, the hypothesis predicts that extents demarcated by familiar objects should be perceived as more distant and, assuming the sizedistance invariance hypothesis, as longer than extents demarcated by unfamiliar objects. This prediction was evalu- 
ated in Experiment 3. In effect, Experiment 3 duplicated Carlson and Tassone's experiment, except that the familiar and unfamiliar objects were substituted by extents, one demarcated by familiar objects and the other by unfamiliar objects.

\section{EXPERIMENT 3}

The midpoints of the two intervals, each $10 \mathrm{~m}$ long, were located $44^{\circ}$ apart and $160 \mathrm{~m}$ from the subjects' location. The intervals were orthogonal to the subjects' line of sight. One interval was demarcated by the two person markers, both approximately $170 \mathrm{~cm}$ tall and of similar weight, and the other by two upright white boards, $30 \mathrm{~cm}$ wide and $170 \mathrm{~cm}$ high. The left/right locations of the two intervals were controlled across subjects. The subjects were 30 volunteer undergraduate students, none of whom had participated in the previous experiments. In the first phase of the experiment, all subjects were asked to nominate which of the two intervals appeared longer/shorter. In the second phase of the experiment, 22 of the 30 subjects were asked to report which interval appeared farther away. For the remaining 8 subjects, this phase of the experiment was similar to the third phase of Experiment 2; the test objects were a single person and a single board, located $44^{\circ}$ apart and $160 \mathrm{~m}$ from the subject's location, and the subjects were required to state which of the two objects appeared farther away.

Of the 30 subjects, 17 subjects nominated the interval demarcated by the person as longer than the interval demarcated by the boards. This proportion (.57) is not significantly different from .5 , indicating that the two intervals were perceived as equal in length. All of the 8 subjects who judged the single person and board targets reported the person to be more distant than the board, thereby confirming Carlson and Tassone's (1971) effect. However, only 12 of the 22 subjects who judged the intervals reported the person interval as being more distant than the board interval. This proportion (.55) is not significantly different from .5 .

The present findings are intriguing. They suggest that Carlson and Tassone's (1971) effect is specific to objects. Familiar size influences the relative size and distance of objects but not the relative lengths or relative distances of exocentric extents.

\section{GENERAL DISCUSSION}

The results of the present investigation indicate that the familiar/unfamiliar characteristic of markers demarcating an exocentric extent do not influence depth-matching judgments (Experiments 1 and 2) or judgments of relative depth (Experiment 2), nor does it influence judgments of the relative lengths and relative egocentric distances of extents presented in subjects' frontoparallel plane (Experiment 3). Additionally, and consistent with Carlson and Tassone's (1971) findings, the results of Experiments 2 and 3 show that familiar size influences judgments of the relative egocentric distances of unfamiliar and familiar objects.

Carlson and Tassone's (1971) hypothesis that at appreciable distances the familiar size of an object alters the subjective distance scale in the direction of increasing perceived distance seems inconsistent with the present findings. If the hypothesis were correct, familiar size should increase not only the perceived distance of a single familiar object but also the perceived distances and the perceived depths and lengths of extents demarcated by familiar objects. From the perspective of Carlson and Tassone's hypothesis, there does not seem to be any plausible theoretical reason for expecting the rescaling effect of familiar size on the subjective distance scale to be specific to perceptions of egocentric distance. ${ }^{2}$

The present findings might be explained in terms of different informational constraints characterizing egocentric and exocentric distance tasks. Specifically, familiar size is only one of the few cues, including absolute motion parallax and oculomotor cues, which potentially determine the apparent distance of an object from an observer. If, as has been claimed (Gogel, 1977), the effectiveness of the major visual distance cues decreases with increasing physical distance, it seems reasonable to suppose that familiar size becomes an increasingly effective source of distance information at increasing physical distances. In a sense, the appreciable viewing distances mimic a cue-reduction situation, and for this reason familiar size is likely to be an important source of distance information. In contrast, the present findings suggest that familiar size information is not an important determinant of spatial judgments of exocentric extents, and this is to be expected given the numerous and redundant cues to perceived relative depth, such as the relative height of the markers in the visual field, texture occlusion, and so forth.

An unresolved issue is the perceptual status of Carlson and Tassone's (1971) effect. A recent proposal (Predebon, 1990) attributes the effect not to the influence of familiar size on perceptions of distance, but to subjects' cognitive beliefs about size-distance relations. According to this hypothesis, the effect occurs if the perspective attitude or bias is elicited by the experimental situation. This attitude refers to the widespread belief among subjects that the apparent size of an object decreases with distance (Carlson, 1960, 1977). Whether or not the perspective bias is elicited in a particular situation depends on a number of factors (see Carlson, 1977), including the distance of the objects, the experimental instruction, and the observer's assessment of the "apparent" size of the objects. Importantly, the hypothesis assumes that at appreciable distances, a familiar object will be experienced as smaller than normal-sized-that is, as being "doll-like" in appearance. Such an assumption is consistent with the claim that at far viewing distances perceived distance underestimates physical distance, which, in agreement with the size-distance invariance hypothesis, produces a corresponding underestimation of the perceived size of 
familiar objects (Gogel \& Da Silva, 1987). The hypothesis claims that Carlson and Tassone's (1971) effect arises because subjects inappropriately compare the doll-like appearance of the familiar object on the one hand with the perceived physical size of the unfamiliar target on the other, and for this reason report the familiar target as being smaller in apparent size. Furthermore, since the familiar and unfamiliar objects are physically equal in size and are perceived as such, the impression of a smallerthan-normal familiar object will elicit the perspective bias, and consequently, the familiar object will be reported as being more distant than the unfamiliar object. Note that this distance judgment cannot be construed as a perceptual response to distance; rather, it is a cognitive response based on observers' beliefs about size-distance relations.

Although the present findings are consistent with the cognitive hypothesis of Carlson and Tassone's (1971) effect, paradoxically the results of Experiment 3 might seem to pose problems for the hypothesis. If at appreciable distances a person is experienced as being doll-like in appearance and, in agreement with the perspective attitude, is reported to be more distant than an equally sized unfamiliar object, why was the interval demarcated by persons not judged to be more distant than the interval demarcated by the boards? According to the cognitive hypothesis, however, the perspective bias will be elicited only if the interval between the persons appears to be shorter than the interval between the boards. Since there are no plausible reasons for subjects to misperceive the relative extents of the two physically equally long intervals viewed under naturalistic conditions, they will be reported to be equal in length and at the same distance. Even though the persons are experienced as doll-like in appearance, such an impression is compatible with subjects' beliefs about size-distance relations on the one hand, and with their spatial perceptions on the other: The dolllike impression of the persons merely confirms subjects' perspective belief that a familiar object appears to be smaller than normal at appreciable distances, an impression that is consistent with the perception that both intervals are at the same appreciable distance.

In summary, the present findings indicate that at appreciable distances familiar size does not affect spatial judgments of exocentric extents, although it does influence judgments of the relative egocentric distances of objects. Taken together, these findings are consistent with the claim that Carlson and Tassone's (1971) effect is likely to reflect the influence of nonperceptual or cognitive factors on subjects' spatial judgments rather than an effect of familiar size on perceptions of extents.

\section{REFERENCES}

CARLSON, V. R. (1960). Overestimation in size constancy judgments. American Joumal of Psychology, 73, 199-213.

CARLSON, V. R. (1977). Instructions and perceptual constancy judgments. In W. Epstein (Ed.), Stability and constancy in visual perception: Mechanisms and processes (pp. 217-254). New York: Wiley.

CARLson, V. R., \& TAssone, E. P. (1971). Familiar versus unfamiliar size: A theoretical derivation and test. Journal of Experimental Psychology, 87, 109-115.

Fillengaum, S., Schiffman, H. R., \& Butcher, J. (1965). Perception of off-size versions of a familiar object under conditions of rich information. Journal of Experimental Psychology, 69, 298-303.

GiBson, J. J. (1950). The perception of the visual world. Boston: Houghton Mifflin.

GoGEL, W. C. (1973). The organization of perceived space: II. Consequences of perceptual interactions. Psychologische Forschung, 33, $1-24$.

GoGEL, W. C. (1977). The metric of visual space. In W. Epstein (Ed.), Stability and constancy in visual perception: Mechanisms and processes (pp. 129-181). New York: Wiley.

GoGEL, W. C., \& DA SiLvA, J. A. (1987). A two-process theory of the response to size and distance. Perception \& Psychophysics, 41, 220-238.

ITTELSON, W. H. (1960). Visual space perception. New York: SpringerVerlag.

Predebon, J. (1979a). Effect of familiar size on judgments of relative size and distance. Perceptual \& Motor Skills, 48, 1211-1214.

Predebon, J. (1979b). The role of familiar size in spatial judgments under natural viewing conditions. Perceptual \& Motor Skills, 48, 171-176.

Predebon, J. (1990). Relative distance judgments of familiar and unfamiliar objects viewed under representatively natural conditions. Perception \& Psychophysics, 47, 342-348.

Rogers, S. P., \& GoGEL, W. C. (1975). Relation between judged and physical distance in multicue conditions as a function of instructions and tasks. Perceptual \& Motor Skills, 41, 171-178.

SEDGWICK, H. A. (1986). Space perception. In K. R. Boff, L. Kaufman, \& J. P. Thomas (Eds.), Handbook of perception and human performance: Vol 1. Sensory processes and perception (pp. 21.121.57). New York: Wiley.

\section{NOTES}

1. The 40-m starting position was chosen for a pragmatic reason. Since the points of subjective equality for each of the three depth intervals at each of the three egocentric distances were unknown, the starting position was set at half the maximum range of the comparison extent $(80 \mathrm{~m})$. I am aware of the problem that the depth-matching errors will include a component attributable to the starting position. However, the issue investigated in this experiment is not the absolute magnitude of the depth errors per se but, rather, the differential effect of marker type on these errors.

2. One reviewer noted that since the characteristic size of persons is not well defined, the effects of familiar size might be more evident if objects of more narrowly defined familiar size were tested. This is unlikely to explain the null findings of the depth-matching tasks, however. If this were the case, judgments of the relative size and relative distance of the single person and single board also should have been unaffected by familiar size. 